\title{
An Assessment of Crosstalk between Serotonin and Dopamine Systems after Escitalopram Treatment Using LC-MS Technique with Ion-trap Analyser
}

\author{
PRATHYUSHA S. NALLELLA, NITESH KUMAR1 AND S. MOORKOTH* \\ Department of Pharmaceutical Quality Assurance, ${ }^{1}$ Department of Pharmacology, Manipal College of Pharmaceutical Sciences \\ Manipal Academy of Higher Education, Manipal-576 104, India
}

Prathyusha et al.: Assessment of cross talk in neurotransmitter systems by LCMS

\begin{abstract}
The present work was aimed at developing and validating a highly sensitive analytical method to estimate the levels of monoamines from mice brain and to apply the developed method to assess the effect of escitalopram treatment on the levels of serotonin, dopamine and noradrenaline in the mouse brain. Liquid chromatography-mass spectroscopy was optimized to achieve separation of selected neurotransmitters. Method validation was performed as per the ICH guidelines. Extraction of the neurotransmitters from mice brain tissue was optimized to achieve highest recovery. In vivo study involved behavioural profile assessment after $7 \mathrm{~d}$ pre-treatment with escitalopram and correlating the results with monoamines levels in their brain homogenate. Behavioural profiles showed a significant $(p<0.05)$ change in all tested parameters. These values were in agreement with the significant rise in serotonin levels $(\mathbf{p}<0.05)$ seen on escitalopram treatment compared to vehicle control. Along with the elevation in serotonin levels, a significant increase in levels of dopamine and a mild increase in noradrenaline levels was observed due to the possible crosstalk between the neurotransmission pathways. Liquid chromatography-mass spectroscopy method was developed and validated for estimation of brain neurotransmitters which revealed a crosstalk resulting in the elevation in levels of various monoamines after escitalopram treatment.
\end{abstract}

Key words: Antidepressants, depressive disorder, escitalopram, ESI mass spectrometry, liquid chromatography, neurotransmitters, neurotransmitter uptake inhibitor

Dopamine, noradrenaline and serotonin are relevant markers for depression according to monoamine hypothesis ${ }^{[1-3]}$. Depression is a common psychiatric disorder characterised by depressed mood, decreased or loss of interest or pleasure, insomnia, feeling of guilt, significant weight loss or weight gain ${ }^{[4]}$. Unknown aetiology and unavailability of relevant markers make the diagnosis and prognosis challenging. Monoamine hypothesis was proposed by Schildkraut in 1965 and is considered to be the most important theories of depression ${ }^{[5-7]}$. As per this hypothesis, depression occurs due to a functional deficiency of monoaminergic activity in the brain namely noradrenaline, dopamine and serotonin ${ }^{[8]}$. Monoaminergic system is very important in regulating mood, motivation, and alertness; any abnormality in this system can lead to depression. Reports are suggesting a crosstalk among the biosynthetic pathways of monoamines during the

*Address for correspondence E-mail: manjula59@gmail.com treatment of depression by the drugs acting through monoamine pathway ${ }^{[9-12]}$. Selective serotonin reuptake inhibitors (SSRIs), as a class of antidepressants are known to change the dopaminergic and noradrenergic firing levels in different parts of the brain along with its mainstay effect of elevating the serotonin levels. This change in neurotransmission pattern depends on the duration of treatment of SSRIs ${ }^{[10,13-15]}$. The acute and chronic treatment of SSRIs showed an increase in serotonin and dopamine in limbic system. Noradrenaline showed no change after acute treatment, but showed a decrease in their levels on chronic treatment with

This is an open access article distributed under the terms of the Creative Commons Attribution-NonCommercial-ShareAlike 3.0 License, which allows others to remix, tweak, and build upon the work non-commercially, as long as the author is credited and the new creations are licensed under the identical terms

Accepted 23 June 2020

Revised 26 May 2020

Received 12 December 2020 Indian J Pharm Sci 2020;82(4):612-621 
SSRIs ${ }^{[13]}$. However reports are scarce indicating the levels of monoamines in the whole brain during a subacute treatment by SSRIs such as escitalopram. This requires a sensitive and reproducible bioanalytical method for simultaneous estimation of noradrenaline, dopamine and serotonin.

In recent years, several analytical techniques such as chromatography, chemiluminescence, colorimetry and mass spectrometry have been proposed and reported to quantify the concentration of neurotransmitters from different biological systems. These include HPLC-FLD (fluorescence detection), HPLC-ECD (electrochemical detection), UHPLC-atmospheric pressure ionisationmass spectrometry (API-MS), UHPLC-electro spray ionisation-mass spectrometry (ESI-MS), and GC-MS techniques ${ }^{[16-22]}$. All these methods have compromised at one place or the other in achieving the required sensitivity and selectivity to analyse minute quantities of neurotransmitters from a complex biological matrix such as the brain homogenate. These problems include noisy signals ${ }^{[23-26]}$, need for tedious derivatization procedure $^{[27-28]}$ and requirement of extensive sample clean up and pre-treatment ${ }^{[29-36]}$.

The present study was undertaken with an aim to develop a simple and sensitive liquid chromatographymass spectroscopy (LC-MS) technique with good selectivity that is devoid of laborious derivatization steps and costly sample extraction/clean up procedures to estimate the levels of monoamines from the mice brain. Use of ion-trap as the mass analyzer has the ability to provide better selectivity and sensitivity. The developed method was applied to evaluate the crosstalk in monoamines in acute depression like model in mice.

\section{MATERIALS AND METHODS}

Noradrenaline ( $>98 \%$ purity), serotonin hydrochloride ( $>98 \%$ purity), dopamine hydrochloride ( $>98.5$ $\%$ purity) and isoprenaline ( $>98 \%$ purity) were all procured from Sigma Aldrich. Acetonitrile (LC-MS grade, Biosolve Chimie, France), Milli Q water (Ultra-clear, Siemens), formic acid and ethanol (Finar Chemicals, India) were used in this study.

A Thermo Scientific (Massachusetts, USA) LC-MS with Dionex Ultimate 3000 liquid chromatograph interfaced with a linear ion trap analyser and an electron spray ionization source was used. $\mathrm{MS}^{\mathrm{n}}$ and chromatographic method development were performed using LTQ XL (Massachusetts, USA) and Chromeleon (Massachusetts, USA) software, respectively. Batch analysis was done using the XCalibur software (Massachusetts, USA) and for quantification LC Quan (Massachusetts, USA) software was used. Open field test chambers developed by PERSPEX were used to study the depression behavior.

\section{Experimental animals:}

C57BL/6 mice (20-25 g) were procured from the Central Animal Research Facility (CARF) of Manipal Academy of Higher Education, Manipal. These mice were acclimatized to the new environment for $7 \mathrm{~d}$ before initiating the experiment. They were housed in conditions of controlled temperature $\left(23 \pm 2^{\circ}\right)$ and humidity $(50 \pm 5 \%)$ with a $12 \mathrm{~h}$ light-dark cycle. Mice were kept in sterilized propylene cages containing sterile husk and were provided with food and water. The experimental protocol was approved by the Institutional Animal Ethics Committee (IAEC), Kasturba Medical College, Manipal Academy of Higher Education, Manipal, constituted as per the guidelines laid by the Committee for the Purpose of Control and Supervision of Experiments on Animals (CPCSEA). IAEC number: Reg No.94/PO/ReBi/S/99/CPCSEA dated 28/04/2017.

\section{Preparation of quality control and calibration standards:}

Calibration standards in brain homogenate $(80,160$, 800, 1600, 3200, 6400, 9600, 12 800, $16000 \mathrm{ng} / \mathrm{ml})$ were prepared by spiking blank brain homogenate with $10 \mu \mathrm{l}$ of drug solution. Four quality control levels were established as $160 \mathrm{ng} / \mathrm{ml}$ (LLOQC), $200 \mathrm{ng} / \mathrm{ml}$ (LQC), $8000 \mathrm{ng} / \mathrm{ml}$ (MQC), $14400 \mathrm{ng} / \mathrm{ml}$ (HQC). These QC standards were used to investigate intra and inter run variations. 2

\section{Optimized chromatographic conditions:}

After performing trials to achieve sufficient separation and peak shapes, the following optimal conditions were arrived at, chromatographic mode was isocratic reverse phase, stationary phase was Phenomenex RP C18 $(150 \times 4.6 \mathrm{~mm}, 5 \mu \mathrm{m})$, mobile phase was $0.1 \%$ formic acid in water:0.1 \% formic acid in acetonitrile (80:20\% $\mathrm{v} / \mathrm{v}$ ), rinsing solution was acetonitrile:methanol:water $(70: 20: 10 \% \mathrm{v} / \mathrm{v})$, run time of $10 \mathrm{~min}$, flow rate of $0.5 \mathrm{ml} / \mathrm{min}$, injection volume of $20 \mu \mathrm{l}$, column temperature of $35^{\circ}$ and auto sampler temperature of $4^{\circ}$. Retention time for the analytes under the above conditions was found to be $3.90,4.04,4.34$ and $4.20 \mathrm{~min}$ for noradrenaline, dopamine, serotonin and 
isoprenaline (internal standard), respectively and the chromatograms under these optimal conditions is shown in fig. 1.

\section{Optimized mass spectrometric conditions:}

Mass spectrometric data was obtained in positive mode with electrospray ionization (ESI) and selected ion monitoring (SIM). Optimized mass spectrometric parameters were as follows, heater temperature of $350^{\circ}$, sheath gas flow rate of 37 psi, auxillary gas flow rate at 7 psi, sweep gas flow rate at 7 psi, capillary temperature of $275^{\circ}$, capillary voltage of $3.5 \mathrm{kV}$, tube lens at $79 \mathrm{~V}$. Details of SIM scan such as base peak $(\mathrm{m} / \mathrm{z})$, collision potential (v) and collision cell exit potential (v) for the analytes and the internal standard were, dopamine (153.5-154.5, 23, 13), norepinephrine (169.5-170.5, 17, $15)$, serotonin $(176.5-177.5,28,15)$ and isoprenaline $(211.5-212.5,35,18)$.

\section{Optimized extraction technique:}

Trials were performed to achieve maximum recovery of analytes from the brain tissue. The optimized extraction procedure was, weighed brain samples were homogenized in ice cold $2 \%$ formic acid in water $(15 \mathrm{ml} / \mathrm{g})$ and centrifuged for $60 \mathrm{~min}$ at $12000 \mathrm{rpm}$ at $4^{\circ}$. Internal standard was then added to the supernatant. Protein precipitation was achieved by the addition of acetonitrile. The supernatant mixture was then centrifuged for $10 \mathrm{~min}$ at $12000 \mathrm{rpm}$. Resulting supernatant was evaporated to dryness at $45^{\circ}$ for $1 \mathrm{~h}$ in a nitrogen evaporator. The residue obtained was then reconstituted with $200 \mu 1$ mobile phase.

\section{Validation of the developed method:}

The developed analytical method was validated for system suitability, sensitivity, selectivity, carryover check, linearity, accuracy and precision as per the FDA guidance for industry ${ }^{[37]}$. System suitability test was performed before each new sequence. Five injections were given from the system suitability solution and \% $\mathrm{CV}$ was calculated for the peak area ratio and for the retention times of the analytes. Selectivity was assessed by injecting extracted blank and compared it with the response of extracted LOQ QC samples. Sensitivity of the method represented by LOQ QC was evaluated for the \% CV using 6 independent injections of the same. Linearity of the method was evaluated on nine non-zero standards covering the range. Correlation coefficient was calculated from the linearity curve. Accuracy and precision were calculated in 6 replicates on the calibration standards and QCs. Extraction recovery of the analytes was evaluated by comparing the mean peak area of all three QC samples to the mean peak area of neat reference solutions.

Stability studies were carried out to ensure that the selectedanalytes werestableunderrealtimeexperimental conditions. Stability of the selected neurotransmitters in the matrix (mice brain homogenate) was evaluated at bench top ( 1 to $8 \mathrm{~h}$, at room temperature), 3 freeze and thaw cycles $\left(-80^{\circ}\right)$ and in the autosampler $\left(4^{\circ}\right.$, from 6 to $48 \mathrm{~h}$ ). The long term stability in matrix during storage at $-80^{\circ}$ was assessed for up to $180 \mathrm{~d}$. For all the studies 6 replicates of LQC and HQC were used and the \% change at each QC level was calculated.

\section{Evaluation of crosstalk between neurotransmitter pathways after escitalopram treatment:}

To assess the crosstalk, pretreatment with escitalopram (SSRI) was given and behavioral profiles were assessed. These behavioral parameters were correlated with the monoamines levels in the brain homogenate. Escitalopram $10 \mathrm{mg} / \mathrm{kg}$ po was used as the standard drug. All doses were freshly prepared in $0.25 \%$ carboxymethyl cellulose (CMC) and animals were pre-treated at a dosing volume of $10 \mathrm{ml} / \mathrm{kg}, 1 \mathrm{~h}$ before inducing stress daily for $7 \mathrm{~d}$ and conducting the behavioral study on $\mathrm{d} 1$ and $\mathrm{d}$ 7. After acclimatization for $7 \mathrm{~d}$, the animals were randomized into 2 groups consisting of 6 animals in each group, and body weights were recorded. Group I, the normal control consisted of 6 animals and was treated with vehicle $0.25 \% \mathrm{v} / \mathrm{v}$ CMC. Group II, the standard group consisted of 6 animals treated with $10 \mathrm{mg} / \mathrm{kg}$ escitalopram. Treatments were given through $\mathrm{d} 1$ to $\mathrm{d} 7$. Depression was assessed in all animals on $\mathrm{d} 1$ and $\mathrm{d} 7$ using behavioral tests such as open field test (OFT), forced swim test (FST) and tail suspension test (TST). On d 7, after the behavioral studies, animals were sacrificed to isolate the brain, which was homogenized in $0.1 \%$ formic acid. Dopamine, noradrenaline and serotonin levels in the brain homogenate were estimated using the developed LC-MS method. The levels of these markers were then correlated with depression behavior.

OFT was performed by placing each animal in the central square of the OFT apparatus, a glass/plastic chamber consisting of 9 squares $(30 \times 30 \mathrm{~cm}$ each $)$ and observed for a total period of $8 \mathrm{~min}(1 \mathrm{~min}$ acclimatization and 7-min testing) for parameters such as line crossing, rearing and grooming after 1,3 and $6 \mathrm{~h}$ of per oral dosing ${ }^{[38]}$. FST was performed by placing each mouse 
individually in a glass cylindrical tank (height: $80 \mathrm{~cm}$, diameter: $30 \mathrm{~cm}$ ) filled with water $\left(23-25^{\circ}\right)$ up to a height of $40 \mathrm{~cm}$, and observed for parameters such as immobility time, swimming time, and struggling time after 1,3 and $6 \mathrm{~h}$ post dosing. Each session lasted $6 \min ^{[38]}$. TST, in which mice were suspended on the edge of a table $50 \mathrm{~cm}$ above the floor by adhesive tape placed approximately $1 \mathrm{~cm}$ from the tip of the tail. The immobility time was measured as per the reported procedure for $6 \mathrm{~min}^{[38]}$. The test was conducted every day 1,3 and $6 \mathrm{~h}$ after treatment until $\mathrm{d} 7$.

On completion of the study, mice were sacrificed by euthanasia with anaesthetic ether. The head was separated from the body and scalp was cut to expose the skull. Postero-anterior cut was made in the midline of the skull, followed by medio-lateral cut above the eyes. The flap formed was cut gently and the brain was isolated by carefully cutting meninges and cranial nerves ${ }^{[39]}$. Brain was stored at $-80^{\circ}$. Before analysis, the brain was thawed unassisted.

\section{Statistical analysis:}

The data obtained were statistically analyzed using GraphPad Prism version 5.0 software. Statistical comparison was done using two-way ANOVA followed by Bonferroni post hoc test. All results were expressed as mean \pm SEM. $\mathrm{P}<0.05$ was considered statistically significant at $95 \%$ confidence interval.

\section{RESULTS AND DISCUSSION}

Neurotransmitters such as dopamine, noradrenaline, and serotonin are polar in nature with $\mathrm{pKa}$ values of 8.58, 9.31 and 8.93, respectively. These compounds have more retention on hydrophilic lipophilic columns (HILIC). As these columns are very expensive, C18 column was tried by altering the ratio of buffer $(0.1 \%$ formic acid in water). Column selection was based on the $\mathrm{pKa}$ and $\log \mathrm{P}$ values of the analytes. Hypersil Gold C18 $(100 \times 4.6 \mathrm{~mm} 3 \mu)$ column was used for trials on which the peak shape was not good. Better peak shape was observed with Phenomenex RP C18 $(150 \times 4.6 \mathrm{~mm}$, $5 \mu \mathrm{m})$ column. Choice of buffer is limited in LC-MS and hence the trials with mobile phase were limited to $0.1 \%$ formic acid in water and $0.1 \%$ formic acid in acetonitrile. Use of formic acid in the buffer helped to keep the drug in ionized state. Varying proportions of $0.1 \%$ acetonitrile and water such as 80:20, 70:30 and $60: 40 \% \mathrm{v} / \mathrm{v}$ were studied for the proper selection of ratio of the mobile phase. The flow rate of $0.2,0.3$ and $0.5 \mathrm{ml} / \mathrm{min}$ were used, and chromatograms were recorded. The flow rate of $0.5 \mathrm{ml} / \mathrm{min}$ was found to be optimal as the retention time was reduced from $10 \mathrm{~min}$ to $4.5 \mathrm{~min}$. Trials with different column oven temperatures such as 30,35 and $40^{\circ}$ were attempted but no predictable changes were observed. So, the temperature was set at $35^{\circ}$. Selection of internal standard was based on the physicochemical properties of the drugs. While optimizing the extraction technique, the focus was to achieve better specificity with consistent recovery. Protein precipitation technique using acetonitrile was found to be the most suitable extraction technique. The chromatogram obtained using the optimized extraction conditions is presented in fig. 1.

Validation of the method was performed for parameters such as system suitability, selectivity, carryover, sensitivity, linearity, accuracy and precision as per FDA guidelines $^{[37]}$. The system suitability was assessed for peak area and retention time. Each day 6 injections were given. The mean $\% \mathrm{CV}$ for 6 days was found to be $0.85,1.20,0.63$ and $0.98 \%$ for dopamine, noradrenaline, serotonin and isoprenaline, respectively for peak area. The mean $\% \mathrm{CV}$ for retention time was found to be $1.06,1.50,0.52$ and $0.65 \%$ respectively, for the above analytes. These results were less than $2 \%$, which was the acceptance criteria for system suitability. The selectivity study showed that the developed method was highly selective with no interference from the matrix or from the internal standard. The $\% \mathrm{CV}$ at LLOQ for dopamine, noradrenaline and serotonin was found to be $18.8,11.7$ and $17.18 \%$ signifying that it is within acceptance criteria, which is $<20 \%$. The carryover study demonstrated that there is no analyte peak detected in the blank. This shows that the elution is complete with the selected mobile phase. The linearity study proved that all the analytes were linear in the range of $160 \mathrm{ng} / \mathrm{ml}$ to $14400 \mathrm{ng} / \mathrm{ml}$ with the regression coefficient of $0.998,0.999$ and 0.992 respectively, for dopamine, noradrenaline and serotonin. Linearity of the analytes is depicted in the fig. 2. The intra-day and inter day precision of the method was determined by calculating the $\% \mathrm{CV}$. All values were found to be $<15 \% \mathrm{CV}$ and were acceptable. Accuracy of the method was determined by calculating the \% nominal concentration, which was found to be in the range of $85.80-113.90 \%$ for dopamine, 87.01-102.49\% for noradrenaline and 92.67-103.52\% for serotonin. All these values were within the acceptance range of $85-115 \%$. The sensitivity study conducted at LLOQ level of $160 \mathrm{ng} / \mathrm{ml}$ showed a \% CV of $13.49,14.74$ and $7.08 \%$ for dopamine, noradrenaline and serotonin, respectively suggesting the method was sensitive. To 
validate the extraction efficiency of the developed extraction procedure, recovery studies were conducted. Accordingly, the method showed acceptable recovery with all values within the acceptable limits of 85 $-115 \%$. A summary of validation results is provided in Table 1.
Stability studies demonstrated that all the analytes were stable at all the tested conditions (Table 2) and the \% change observed were well within the acceptable limits of $\pm 15 \%$. Bench top stability study showed that the maximum \% change at LQC for dopamine, noradrenaline and serotonin after $8 \mathrm{~h}$ at room temperature was 9.59 ,

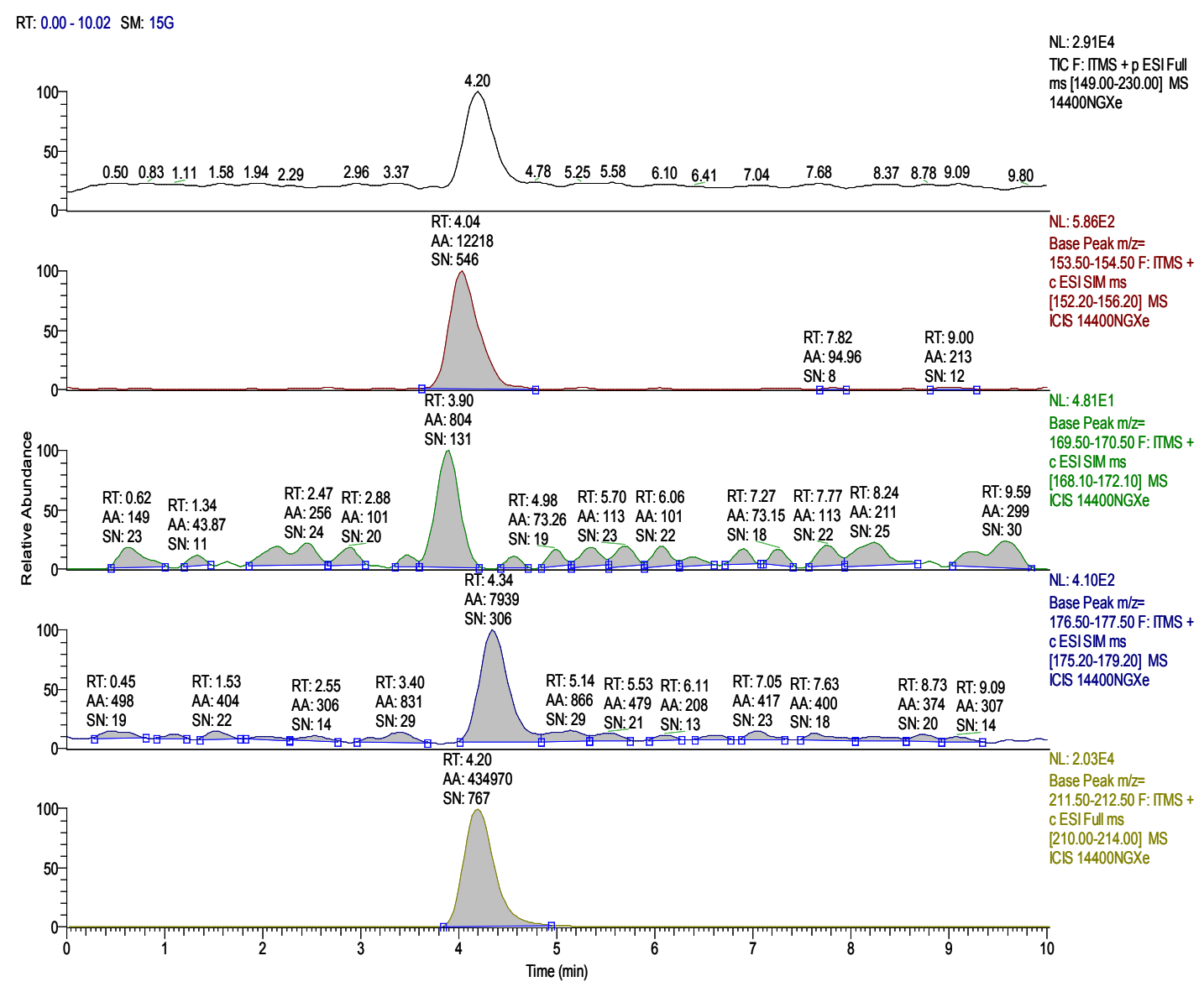

Fig. 1: LC-MS chromatogram of neurotransmitters obtained under optimized conditions

Retention time (RT)- 4.34 (serotonin), 3.90 (noradrenaline), 4.04 (dopamine), 4.20 (isoprenaline- internal standard); Unintegrated chromatogram at RT 4.20 represents the total ion chromatogram (TIC) for the mass range 149 to 23

TABLE 1: METHOD PERFORMANCE SPECIFICATIONS FOR DOPAMINE, NORADRENALINE AND SEROTONIN

\begin{tabular}{|c|c|c|c|c|c|c|c|}
\hline \multirow{3}{*}{ Neurotransmitter } & \multicolumn{7}{|c|}{ Performance specifications } \\
\hline & \multirow{2}{*}{$\begin{array}{c}\text { Calibration range } \\
(\mu \mathrm{g} / \mathrm{ml})\end{array}$} & \multirow{2}{*}{$\begin{array}{l}\text { QC levels } \\
(\mu \mathrm{g} / \mathrm{ml})\end{array}$} & \multicolumn{2}{|c|}{ Accuracy (\%) } & \multicolumn{2}{|c|}{ Precision (\% CV) } & \multirow{2}{*}{$\begin{array}{l}\text { Percent } \\
\text { recovery }\end{array}$} \\
\hline & & & Inter & Intra & Inter & Intra & \\
\hline \multirow{4}{*}{ Dopamine } & \multirow{4}{*}{$0.16-14.4$} & LLOQ(0.16) & 88.81 & 85.80 & 4.64 & 5.63 & - \\
\hline & & LQC (0.20) & 98.40 & 99.95 & 0.51 & 1.02 & 87.90 \\
\hline & & MQC (8.00) & 92.54 & 105.07 & 1.68 & 2.06 & 101.04 \\
\hline & & HQC (14.40) & 101.87 & 113.90 & 10.52 & 0.15 & 90.69 \\
\hline \multirow{4}{*}{ Noradrenaline } & \multirow{4}{*}{$0.16-14.4$} & LLOQ(0.16) & 89.74 & 87.01 & 4.52 & 2.26 & - \\
\hline & & LQC (0.20) & 99.87 & 102.48 & 6.44 & 1.78 & 89.43 \\
\hline & & MQC (8.00) & 102.49 & 101.00 & 16.24 & 3.39 & 98.13 \\
\hline & & HQC (14.40) & 100.69 & 101.73 & 10.18 & 8.32 & 102.69 \\
\hline \multirow{4}{*}{ Serotonin } & \multirow{4}{*}{$0.16-14.4$} & LLOQ(0.16) & 103.52 & 93.80 & 2.40 & 8.34 & - \\
\hline & & LQC (0.20) & 92.67 & 95.48 & 14.27 & 0.79 & 96.29 \\
\hline & & MQC (8.00) & 102.18 & 99.52 & 3.39 & 1.89 & 97.23 \\
\hline & & HQC (14.40) & 101.59 & 101.23 & 8.34 & 2.18 & 102.34 \\
\hline
\end{tabular}

LLOQ: lower limit of quantification, LQC: low quality control, MQC: medium quality control, HQC: high quality control 

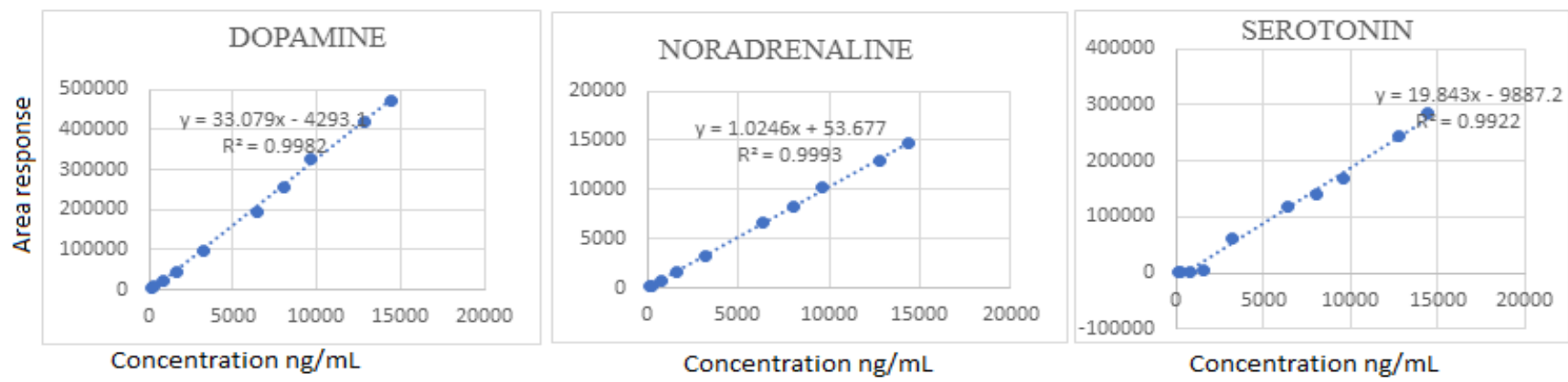

Fig. 2: Linearity plot of dopamine, noradrenaline and serotonin

TABLE 2: BENCH TOP, FREEZE-THAW, AUTOSAMPLER AND LONG TERM (MATRIX) STABILITY DATA FOR DOPAMINE, NORADRENALINE AND SEROTONIN

\begin{tabular}{|c|c|c|c|c|c|c|}
\hline \multirow{2}{*}{ Time points } & \multicolumn{3}{|c|}{$\%$ change at LQC } & \multicolumn{3}{|c|}{$\%$ change at $\mathrm{HQC}$} \\
\hline & Dopamine & Noradrenaline & Serotonin & Dopamine & Noradrenaline & Serotonin \\
\hline \multicolumn{7}{|c|}{ Bench top stability (in matrix at room temperature, $8 \mathrm{~h}$ ) } \\
\hline $0 \mathrm{~h}$ & 0.00 & 0.00 & 0.00 & 0.00 & 0.00 & 0.00 \\
\hline $0.5 \mathrm{~h}$ & 0.00 & 0.00 & 0.00 & 0.09 & 0.08 & 0.00 \\
\hline $1 \mathrm{~h}$ & 0.00 & 0.02 & 0.00 & 1.21 & 0.21 & 0.00 \\
\hline $1.5 \mathrm{~h}$ & 1.44 & 1.18 & 0.55 & 1.10 & 1.13 & 0.49 \\
\hline $2 \mathrm{~h}$ & 1.55 & 1.26 & 0.78 & 1.98 & 1.19 & 0.83 \\
\hline $4 \mathrm{~h}$ & 3.75 & 4.02 & 1.81 & 4.18 & 3.17 & 1.32 \\
\hline $8 \mathrm{~h}$ & 9.59 & 10.35 & 5.18 & 14.14 & 11.76 & 3.87 \\
\hline \multicolumn{7}{|c|}{ Freeze thaw stability (three freeze thaw cycles) } \\
\hline 0 cycle & 0.00 & 0.00 & 0.00 & 0.00 & 0.00 & 0.00 \\
\hline 3 cycles & 2.05 & 3.62 & 1.69 & 2.02 & 2.90 & 1.63 \\
\hline \multicolumn{7}{|c|}{ Autosampler stability $\left(4^{\circ}, 48 \mathrm{~h}\right)$} \\
\hline $0 \mathrm{~h}$ & 0.00 & 0.00 & 0.00 & 0.00 & 0.00 & 0.00 \\
\hline $6 \mathrm{~h}$ & 0.59 & 0.52 & 0.16 & 0.16 & 1.54 & 0.20 \\
\hline $12 \mathrm{~h}$ & 1.39 & 0.61 & 0.14 & 0.64 & 2.33 & 0.33 \\
\hline $24 \mathrm{~h}$ & 1.25 & 0.59 & 0.24 & 1.55 & 3.85 & 0.29 \\
\hline $48 \mathrm{~h}$ & 1.85 & 0.71 & 0.23 & 1.23 & 3.81 & 0.31 \\
\hline \multicolumn{7}{|c|}{ Long term stability (matrix: mice brain, $-80^{\circ}, 180 \mathrm{~d}$ ) } \\
\hline $0 \mathrm{~d}$ & 0.00 & 0.00 & 0.00 & 0.00 & 0.00 & 0.00 \\
\hline $7 \mathrm{~d}$ & 1.13 & 0.28 & 0.14 & 0.26 & 0.31 & 0.33 \\
\hline $15 d$ & 2.10 & 1.57 & 0.96 & 1.22 & 1.21 & 0.69 \\
\hline $30 \mathrm{~d}$ & 0.24 & 1.14 & 0.94 & 1.96 & 2.81 & 1.98 \\
\hline $60 d$ & 1.89 & 1.95 & 1.34 & 2.69 & 2.91 & 2.06 \\
\hline $90 \mathrm{~d}$ & 1.70 & 2.41 & 0.74 & 2.22 & 3.34 & 2.57 \\
\hline $180 \mathrm{~d}$ & 1.15 & 3.27 & 1.89 & 2.62 & 3.26 & 2.86 \\
\hline
\end{tabular}

LQC: low quality control, HQC: high quality control

10.35 , and $5.18 \%$, respectively, while that at HQC was $14.4,11.76$, and $3.87 \%$. These values were within the acceptable limits. The stability study also proved that all the selected neurotransmitters are very stable on storage up to $180 \mathrm{~d}$ and also on repeated freeze and thaw up to 3 cycles. Additionally, the stability observed in the autosampler at $4^{\circ}$ up to $48 \mathrm{~h}$ proved the utility of the method for automation.

SSRIs are the first-line drugs for the treatment of depression and anxiety. Earlier it was considered to restore the balance of serotonin in the brain. However, recent reports are indicating its effects on other monoamine levels also, such as dopamine and noradrenaline firing levels in various parts of the brain ${ }^{[5]}$. For instance, fluoxetine, one of the SSRIs, showed an increase in interstitial serotonin levels and dopamine overflow in the prefrontal cortex and hypothalamus with acute treatment ${ }^{[40]}$, while decreasing the dopamine levels in microdialysates from rat striatum ${ }^{[41]}$. Similarly, escitalopram, a potent SSRI, did not show dampening the firing levels of dopaminergic neuron in the ventral tegmental area (VTA) ${ }^{[14]}$ and increase extracellular dopamine levels in the amygdala ${ }^{[42]}$. However, it is not clear what will be the overall effect of Escitalopram in 
serotonin, dopamine and noradrenaline levels in the brain. Thus, the present study was designed to develop an LC-MS method of these 3 neurotransmitters in the brain homogenate and to assess the crosstalk in releasing of noradrenaline and dopamine along with serotonin on treatment with escitalopram. The levels of neurotransmitters were also correlated with the behavioral parameters using FST, TST and OFT parameters.

As expected, an increase in the levels of serotonin was observed after treatment with $10 \mathrm{mg} / \mathrm{kg}$ of escitalopram (Table 3, fig. 4). Along with serotonin, levels of dopamine and noradrenaline also showed an increasing trend with treatment. The levels of dopamine showed 4 times increase in comparison to the untreated control group while serotonin levels were increased by 3 times. Levels of noradrenaline could register only a one-fold increase. Escitalopram showed a significant $(\mathrm{p}<0.05)$ rise in serotonin levels along with dopamine levels in the mice brain compared to vehicle control. However, the increase in noradrenaline levels was not significant $(\mathrm{p}<0.05)$ compared to vehicle control. Reports also indicate variation in the levels of monoamine transmission following variation in dosing duration of SSRIs. As per the available literature, the acute treatment of SSRIs shows only a slight increase in serotonin in the limbic system while registering no change in noradrenaline level and a minor reduction in dopamine levels. The literature reported that chronic treatment with SSRIs showed a prominent increase in dopamine transmission and a reduction in norepinephrine transmission ${ }^{[7]}$. The findings of this study indicated that a short term treatment of escitalopram showed a similar trend to that of the previously reported chronic treatment except for noradrenaline. The current finding can be explained based on the report of Zhou et $a l .{ }^{[9]}$, where they found that fluoxetine inhibited serotonin transporter, thereby increasing the level of serotonin in striatum, which followed the nonselective reuptake of 5-HT by dopamine transporter in striatal dopamine terminals leading to co-release of 5-HT and dopamine. These workers have shown that the elevated
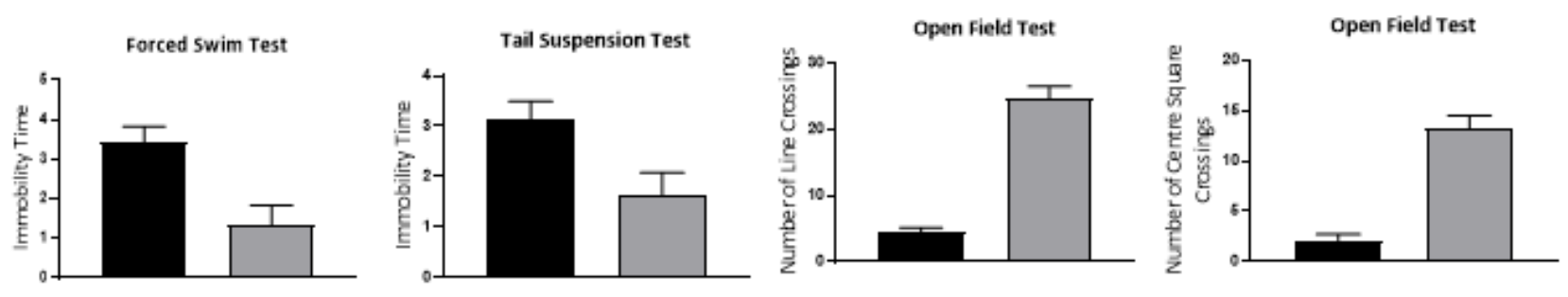

Fig. 3: Results of behavioral studies

Evaluation of immobility time, number of line crossings and number of center square crossings in forced swim test, tail suspension test and open field test after 7 days of treatment with escitalopram. (ロ) Normal group (untreated control); ( $\square$ ) treated group

TABLE 3: ESTIMATED LEVELS OF NEUROTRANSMITTERS IN MICE BRAIN AFTER TREATMENT WITH ESCITALOPRAM

\begin{tabular}{lcc}
\hline & \multicolumn{2}{c}{$\begin{array}{c}\text { Levels of neurotransmitters observed in rat brain after day } 7 \text { of treatment } \\
(\mathrm{ng})\end{array}$} \\
\cline { 2 - 3 } Neurotransmitters & Treated group (treated with standard drug \\
\cline { 2 - 3 } Escitalopram) & $20124.1 \pm 354.60^{\mathrm{a}}$ \\
Noradrenaline & $5310.4 \pm 31.48$ & $1550.5 \pm 17.22$ \\
Serotonin & $817.3 \pm 6.58$ & $17409.6 \pm 152.90^{\mathrm{a}}$ \\
\hline
\end{tabular}

All the values are mean \pm SEM of 6 readings. Values are analysed by unpaired t-test using GraphPad Prism Software. Except noradrenaline all the values are significant at ${ }^{a} p<0.05$. For noradrenaline $p=0.0546$
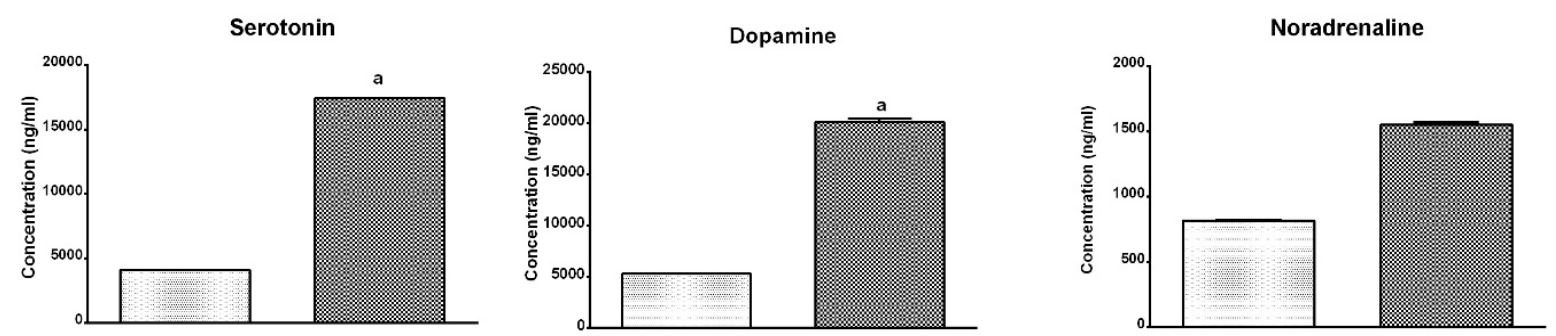

Fig. 4: Levels serotonin, dopamine and noradrenaline after treatment with Escitalopram Levels of neurotransmitters after 7 days of treatment with escitalopram ( $\square$ ) Normal group (untreated control); ( $\square$ ) treated 
5-HT was taken up by DAT into striatal dopamine terminals, which released dopamine and 5-HT together subsequently. A similar report by Muraoka et al. 2018, showed that escitalopram increased dopamine release in amygdala ${ }^{[42]}$.

The increase in levels of these neurotransmitters was then correlated with improvement in behavioral features of depression. The increased levels of monoamine neurotransmitters are linked with the improvement in immobility time in FST and TST in rodents. Kumar et al. correlated the observed anxiolytic activity of Cedrus deodara with the levels of serotonin and noradrenaline in the brain. They have reported a significant reduction in the immobility time in $\mathrm{FST}^{[43]}$. Similar findings were obtained in the present study. The results of behavioral tests were also in corroboration with the results of the above analysis. The increase in the levels of neurotransmitters as estimated by the method were seen to have an inverse relationship with the immobility time in the forced swim test and in the tail suspension test. Similarly, a direct relationship was observed between the estimated levels of neurotransmitters and the number of line crossings/number of center square crossings by mice in the OFT (fig. 3). The observed antidepressant activity was in parallel with the observed levels of the neurotransmitters in the mice brain. This experiment proves that the developed method is able to estimate the levels of neurotransmitters with sufficient confidence to evaluate the antidepressant activity.

In this research work, a sensitive and selective LCMS method for estimating the levels of dopamine, noradrenaline and serotonin from mice brain was developed and validated. The method has advantages of less run time, use of simple isocratic elution technique, no derivatization step and simple sample extraction techniques without compromising on the sensitivity. Application of the method to evaluate crosstalk between monoaminergic pathways showed that a short duration of escitalopram treatment showed an increase in noradrenaline, dopamine and serotonin levels in the whole brain of mice which were in correlation with the decrease in immobility time in FST and TST and improvement in the parameters of OFT compared to normal animals. The change in monoamine levels by escitalopram suggested an existing crosstalk in the monoamines levels even with the limited duration exposure (7 d) of escitalopram.

\section{Acknowledgements:}

All authors have equally contributed to the design of this research work. Bioanalytical method development and validation was mentored by Sudheer Moorkoth and executed by Nellella Sai Prathyusha. Animal experiments and biological evaluation was mentored by Nitesh Kumar and executed by Nellella Sai Prathyusha. All authors have equally contributed in the preparation of the manuscript. Formatting of the manuscript was done by Arya Raveendran from the Department of Pharmaceutical Quality Assurance, Manipal College of Pharmaceutical Sciences, Manipal.

\section{Conflict of interest:}

The authors declare no conflict of interest, financial or otherwise.

\section{REFERENCES}

1. Delgado PL. Depression: The case for a monoamine deficiency. J Clin Psychiatry 2000;61Suppl 6:7-11.

2. Ruhé HG, Mason NS, Schene AH. Mood is indirectly related to serotonin, norepinephrine and dopamine levels in humans: A meta-analysis of monoamine depletion studies. Mol Psychiatry 2007;12(4):331-59.

3. Pan JX, Xia JJ, Deng FL, Liang WW, Wu J, Yin BM, Dong MX, et al. Diagnosis of major depressive disorder based on changes in multiple plasma neurotransmitters: A targeted metabolomics study. Transl Psychiatry 2018;10(8, Suppl 1):1-10.

4. Sharan P. An overview of Indian research in personality disorders. Indian J Psychiatry 2010;52 Suppl1:S250.

5. Schildkraut JJ. The Hypothesis of supporting of affective disorders: Evidence. Am J Psychiatry 1965;122(5):509-22.

6. Marathe SV, D'almeida PL, Virmani G, Bathini P, Alberi L. Effects of monoamines and antidepressants on astrocyte physiology: implications for monoamine hypothesis of depression. J Exp Neurosci 2018; 20(12):1179069518789149.

7. Hamon M, Blier P. Monoamine neurocircuitry in depression and strategies for new treatments. Prog Neuropsychopharmacol Biol Psychiatry 2013;1(45):54-63.

8. Zydroń M, Baranowski J, Białkowski J, Baranowska I. HPLC-FL/ED in the Analysis of Biogenic Amines and their Metabolites in Urine. Sep Sci Technol 2005;40(15):3137-48.

9. Zhou FM, Liang Y, Salas R, Zhang L, De Biasi M, Dani JA. Corelease of dopamine and serotonin from striatal dopamine terminals. Neuron 2005;46(1):65-74.

10. Tritschler L, Gaillard R, Gardier AM, David DJ, Guilloux JP. Consequences of the monoaminergic systems cross-talk in the antidepressant activity. Encephale 2018;44(3):264-73.

11. Blier P. Crosstalk between the norepinephrine and serotonin systems and its role in the antidepressant response. J Psychiatry Neurosci 2001;26(Suppl 1):S3-10.

12. Goddard AW, Ball SG, Martinez J, Robinson MJ, Yang CR, Russell JM, et al. Current perspectives of the roles of the central norepinephrine system in anxiety and depression. Depress Anxiety 2010;27(4):339-50.

13. Jurič DM, Miklič Š, Čarman-Kržan M. Monoaminergic 
neuronal activity up-regulates BDNF synthesis in cultured neonatal rat astrocytes. Brain Res 2006;1108(1):54-62.

14. Dremencov E, El Mansari M, Blier P. Effects of sustained serotonin reuptake inhibition on the firing of dopamine neurons in the rat ventral tegmental area. J Psychiatry Neurosci 2009;34(3):223-229.

15. Prisco S, Esposito E. Differential effects of acute and chronic fluoxetine administration on the spontaneous activity of dopaminergic neurones in the ventral tegmental area. $\mathrm{Br} \mathrm{J}$ Pharmacol 995;116(2):1923-31.

16. Bicker J, Fortuna A, Alves G, Falcão A. Liquid chromatographic methods for the quantification of catecholamines and their metabolites in several biological samples: A review. Anal Chim Acta 2013;768:12-34.

17. Li W, Rossi DT, Fountain ST. Development and validation of a semi-automated method for L -dopa and dopamine in rat plasma using electrospray LC-MS/MS. J Pharm Biomed Anal 2000;24:325-33.

18. De Jong WHA, Wilkens MHLI, De Vries EGE, Kema IP. Automated mass spectrometric analysis of urinary and plasma serotonin. Anal Bioanal Chem 2010;396(7):2609-16.

19. Hows ME, Lacroix L, Heidbreder C, Organ AJ, Shah AJ. High-performance liquid chromatography/tandem mass spectrometric assay for the simultaneous measurement of dopamine, norepinephrine, 5-hydroxytryptamine and cocaine in biological samples. J Neurosci Methods 2004;138(1-2):12332.

20. Kushnir MM, Urry FM, Frank EL, Roberts WL, Shushan B. Analysis of catecholamines in urine by positive-ion electrospray tandem mass spectrometry. Clin Chem 2002;48(2):323-31.

21. Majidano AA, Majidano SA, Alamgir M, Khuhawar MY. Liquid chromatographic determination of dopamine, methyldopa, L-Dopa and tyrosine in pharmaceutical preparations using 2Hydroxynaphthaldehyde as a derivatizing Reagent. Int J Chem Environ Eng 2013;4(3):159-163.

22. Whiting MJ. Simultaneous measurement of urinary metanephrines and catecholamines by liquid chromatography with tandem mass spectrometric detection. Ann Clin Biochem 2009;46(2):129-36.

23. Cai H, Zhu R, Li H. Determination of dansylated monoamine and amino acid neurotransmitters and their metabolites in human plasma by liquid chromatography - electrospray ionization tandem mass spectrometry. Anal Biochem 2010;396(1):103-11.

24. Cheng FC, Shih Y, Liang YJ, Yang LL, Yang CS. New dual electrochemical detector for microbore liquid chromatography determination of dopamine and serotonin in rat striatum dialysates. J Chromatogr B Biomed Sci Appl 1996;682(2):195200.

25. Li XS, Li S, Wynveen P, Mork K, Kellermann G. Development and validation of a specific and sensitive LC-MS/MS method for quantification of urinary catecholamines and application in biological variation studies. Anal Bioanal Chem 2014;406(28):7287-97.

26. Yoshitake $\mathrm{T}$, Kehr J, Yoshitake S, Fujino $\mathrm{K}$, Nohta $\mathrm{H}$, Yamaguchi M. Determination of serotonin, noradrenaline, dopamine and their metabolites in rat brain extracts and microdialysis samples by column liquid chromatography with fluorescence detection following derivatization with benzylamine and 1, 2-diphenylethylenediamine. J. Chromatogr B 2004;807(2):177-183.

27. Fujino K, Yoshitake T, Inoue O, Ibii N, Kehr J, Ishida J, et al. Increased serotonin release in mice frontal cortex and hippocampus induced by acute physiological stressors. Neurosci. Lett 2002;320(1-2):91-5.

28. Huang F, Li J, Shi H, Wang T, Muhtar W, Du M, et al. Simultaneous quantification of seven hippocampal neurotransmitters in depression mice by LC-MS/MS. J Neurosci Methods 2014;229:8-14.

29. Heidbreder CA, Lacroix L, Atkins AR, Organ AJ, Murray S, West A, et al. Development and application of a sensitive high performance ion-exchange chromatography method for the simultaneous measurement of dopamine, 5-hydroxytryptamine and norepinephrine in microdialysates from the rat brain. $\mathrm{J}$ Neurosci Methods 2001;112(2):135-44.

30. Ji C, Li W, Ren XD, El-Kattan AF, Kozak R, Fountain S, Lepsy C. Diethylation labeling combined with UPLC-MS/ MS for simultaneous determination of a panel of monoamine neurotransmitters in rat prefrontal cortex microdialysates. Anal Chem 2008;80(23):9195-203.

31. Talwar D, Williamson C, McLaughlin A, Gill A, O'Reilly DS. Extraction and separation of urinary catecholamines as their diphenyl boronate complexes using $\mathrm{C} 18$ solid-phase extraction sorbent and high-performance liquid chromatography. J Chromatogr B 2002;769(2):341-9.

32. Törnkvist A, Sjöberg PJ, Markides KE, Bergquist J. Analysis of catecholamines and related substances using porous graphitic carbon as separation media in liquid chromatography-tandem mass spectrometry. J Chromatogr B 2004;801(2):323-9.

33. Peitzsch M, Pelzel D, Glöckner S, Prejbisz A, Fassnacht M, Beuschlein F, et al. Simultaneous liquid chromatography tandem mass spectrometric determination of urinary free metanephrines and catecholamines, with comparisons of free and deconjugated metabolites. Clinica Chimica Acta 2013;418:50-8.

34. Carrera V, Sabater E, Vilanova E, Sogorb MA. A simple and rapid HPLC-MS method for the simultaneous determination of epinephrine, norepinephrine, dopamine and 5-hydroxytryptamine: Application to the secretion of bovine chromaffin cell cultures. J Chromatogr B 2007;847(2):88-94.

35. Gill M, Kinra M, Rai A, Chamallamudi MR, Kumar N. Evaluation of antidepressant activity of methanolic extract of Saraca asoca bark in a chronic unpredictable mild stress model. Neuroreport 2018;29(2):134-40.

36. Moriarty M, Lee A, O'Connell B, Kelleher A, Keeley H, Furey A. Development of an LC-MS/MS method for the analysis of serotonin and related compounds in urine and the identification of a potential biomarker for attention deficit hyperactivity/ hyperkinetic disorder. Anal Bioanal Chem 2011;401(8):248193.

37. Food and Drug administration. (2018). Bioanalytical Method Validation Guidance for Industry. Rockville, MD: Food and drug administration. Available from https://www.fda.gov/files/ drugs/published/Bioanalytical-Method-Validation-Guidancefor-Industry.pdf

38. Pratap SR, Ritesh J, Rahul M, Prashant T. Antidepressant activity of hydroalcoholic extract of Zingiber officinale. Int Res J Pharm 2012;3:149-151.

39. Kim TH, Choi J, Kim HG, Kim HR. Quantification of neurotransmitters in mouse brain tissue by using liquid chromatography coupled electrospray tandem mass spectrometry. J Anal Methods Chem 2014;2014:11.

40. Koch S, Perry KW, Nelson DL, Conway RG, Threlkeld PG, Bymaster FP. R-fluoxetine increases extracellular DA, NE, as well as 5-HT in rat prefrontal cortex and hypothalamus: 
An in vivo microdialysis and receptor binding study. Neuropsychopharmacology 2002;27(6):949-959.

41. Clark RN, Ashby CR, Jr. Dewey SL, Ramachandran PV, Strecker RE. Effect of acute and chronic fluoxetine on extracellular dopamine levels in the caudate-putamen and nucleus accumbens of rat. Synapse 1996;23(3):125-131.

42. Muraoka H, Oshibuchi H, Kawano M, Kawano T, Tsutsumi T, Yamada M, et al. Escitalopram attenuates fear stress-induced increase in amygdalar dopamine following methamphetamine- induced sensitisation: Implications of fine-tuning action of selective serotonin reuptake inhibitors on emotional processing. Eur J Pharmacol 2018;834:1-9.

43. Kumar N, Dhayabaran D, Nampoothiri M, Nandakumar $\mathrm{K}$, Puratchikody A, Lalani N, et al. Atypical antidepressant activity of 3, 4-Bis (3, 4-dimethoxyphenyl) furan-2, 5-dione isolated from heart wood of Cedrus deodara, in rodents. Korean J Physiol Pharmacol 2014;18(5):365-369 